\title{
A modified iterative algorithm for finding a common element in Hilbert space
}

\author{
Zhichuan Zhu ${ }^{a, b, *}$, Yanchun Xing ${ }^{a, b}$, Weihua Duan ${ }^{c}$ \\ a School of Statistics, Jilin University of Finance and Economics, Changchun, Jilin 130117, China. \\ ${ }^{b}$ School of Mathematics and Statistics, Northeast Normal University, Changchun, Jilin 130024, China. \\ c Yatai School of Business Administration, Jilin University of Finance and Economics, Changchun, 130117, China.
}

Communicated by Y. H. Yao

\begin{abstract}
In this paper, a modified iterative algorithm for finding a common element of the solutions of a equilibrium problem, the set of fixed points of nonexpansive mappings and the set of solutions of variational inequality problem is constructed in Hilbert spaces, and the strong convergence of the generated iterative sequence to the common element is proved under some mild conditions. The main result proposed in this paper extends and improves some recent results in the literature. (C)2017 all rights reserved.
\end{abstract}

Keywords: Common element, iterative algorithm, Hilbert space, strong convergence. 2010 MSC: 47H10, 55M20.

\section{Introduction}

Let $\mathrm{H}$ be a Hilbert space, and $\mathrm{C}$ be a nonempty closed convex subset of $\mathrm{H}$. Let $\mathrm{f}$ be a bifunction from $C \times C$ to $\Re$, where $\Re$ is the set of real numbers.

The equilibrium problem $F: C \times C \rightarrow \Re$ is to find an element $x \in C$ such that $F(x, y) \geqslant 0$ for all $y \in C$, and the set of such solutions is denoted by EP(F).

Recall that a mapping $f: C \rightarrow C$ is called contractive if there exists a constant $\alpha \in(0,1)$ such that $\|f(x)-f(y)\| \leqslant \alpha\|x-y\|$ for all $x, y \in C$.

A mapping $S: C \rightarrow C$ is called nonexpansive if for all $x, y \in C,\|S x-S y\| \leqslant\|x-y\|$, and the set of fixed points of $S$ is denoted by $\operatorname{Fix}(S)$. It is well-known that if $C$ is bounded closed convex and $S: C \rightarrow C$ is nonexpansive, then $\operatorname{Fix}(S) \neq \phi$.

In 2007, Takahashi and Takahashi [14] introduced an iterative scheme using the viscosity approximation method in a Hilbert space as follows:

$$
\left\{\begin{array}{l}
F\left(y_{n}, u\right)+\frac{1}{r_{n}}\left\langle u-y_{n}, y_{n}-x_{n}\right\rangle \geqslant 0, \quad \forall u \in C, \\
x_{n+1}=\alpha_{n} f\left(x_{n}\right)+\left(1-\alpha_{n}\right) S y_{n},
\end{array}\right.
$$

\footnotetext{
${ }^{*}$ Corresponding author

Email addresses: zhuzcnh@126.com (Zhichuan Zhu), xingyanchun778@163.com (Yanchun Xing), duanweihua@jlufe.edu.cn (Weihua Duan)
}

doi:10.22436/jnsa.010.01.25 
and the strong convergence to a common element $q \in \operatorname{Fix}(S) \cap E P(F)$ was obtained under certain appropriate conditions imposed on $\left\{\alpha_{n}\right\}$ and $\left\{r_{n}\right\}$, where $q=P_{\operatorname{Fix}(S) \cap E P(F)} f(q)$.

Let $A$ be a strongly positive bounded linear operator on $H$, i.e., there exists a constant $\gamma>0$ such that

$$
\langle A x, x\rangle \geqslant \gamma\|x\|^{2} \text { for all } x \in H .
$$

A typical problem is that of minimizing a quadratic function over the set of the fixed points of a nonexpansive mapping on a real Hilbert space $\mathrm{H}$ :

$$
\min _{x \in F(S)} \frac{1}{2}\langle A x, x\rangle-\langle x, b\rangle,
$$

where $\mathrm{b}$ is a given point in $\mathrm{H}$.

In 2006, Marino and $\mathrm{Xu}$ [8] proposed the following iterative algorithm:

$$
x_{n+1}=\left(I-\alpha_{n} A\right) S x_{n}+\alpha_{n} \gamma f\left(x_{n}\right), \quad n \geqslant 0 .
$$

Under some appropriate conditions on parameter $\left\{\alpha_{n}\right\}$, the sequence $\left\{x_{n}\right\}$ generated by (1.1) was proved to converge strongly to the unique solution of the following variational inequality

$$
\langle(A-\gamma f) q, x-q\rangle \geqslant 0, \quad x \in \operatorname{Fix}(S),
$$

which is the optimality condition for the minimization problem

$$
\min _{x \in \operatorname{Fix}(S)} \frac{1}{2}\langle A x, x\rangle-h(x),
$$

where $h$ is a potential function for $\gamma f$ (i.e., $h^{\prime}(x)=\gamma f(x)$ for $x \in H$ ).

In 2007, Plubtieng and Punpaeng [10] introduced and considered the following two iterative schemes for finding a common element of the set of solutions of equilibrium problem and the set of fixed points of a nonexpansive mapping in a Hilbert space:

$$
\begin{cases}F\left(y_{n}, u\right)+\frac{1}{r_{n}}\left\langle u-y_{n}, y_{n}-x_{n}\right\rangle \geqslant 0, & \forall u \in H \\ x_{n}=\alpha_{n} \gamma f\left(x_{n}\right)+\left(1-\alpha_{n} A\right) S y_{n}, & \forall n \geqslant 1,\end{cases}
$$

and

$$
\begin{cases}F\left(y_{n}, u\right)+\frac{1}{r_{n}}\left\langle u-y_{n}, y_{n}-x_{n}\right\rangle \geqslant 0, & \forall u \in H, \\ x_{n+1}=\alpha_{n} \gamma f\left(x_{n}\right)+\left(1-\alpha_{n} A\right) S y_{n}, & \forall n \geqslant 1 .\end{cases}
$$

The sequences $\left\{x_{n}\right\}$ generated by (1.2) and (1.3) were proved to converge strongly to the unique solution of the following variational inequality under some appropriate conditions:

$$
\langle(A-\gamma f) q, x-q\rangle \geqslant 0, \quad \forall x \in \operatorname{Fix}(S) \cap \operatorname{EP}(F),
$$

which is the optimality condition for the minimization problem

$$
\min _{x \in \operatorname{Fix}(S) \cap E P(F)} \frac{1}{2}\langle A x, x\rangle-h(x),
$$

where $h$ is a potential function for $\gamma f$.

For finding a common element of the set of the fixed points of nonexpansive mappings and the set of the solutions to variational inequalities for $\alpha$-cocoercive map, Takahashi and Toyoda proposed the following iterative process in [15]:

$$
x_{n+1}=\alpha_{n} x_{n}+\left(1-\alpha_{n}\right) S P_{C}\left(x_{n}-\lambda_{n} A x_{n}\right),
$$


for every $n=0,1,2, \cdots$, where $A$ is $\alpha$-cocoercive, $x_{0}=x \in C,\left\{\alpha_{n}\right\}$ is a sequence in $(0,1)$, and $\left\{\lambda_{n}\right\}$ is a sequence in $(0,2 \alpha)$. If the set $\operatorname{Fix}(S) \cap \operatorname{VI}(C, A)$ is nonempty, then the sequence $\left\{x_{n}\right\}$ generated by (1.4) was proved to converge weakly to some $q \in \operatorname{Fix}(S) \cap V I(C, A)$.

In 2005, Iiduka and Takahashi [7] proposed another iterative scheme as follows:

$$
x_{n+1}=\alpha_{n} x+\left(1-\alpha_{n}\right) \operatorname{SP} C\left(x_{n}-\lambda_{n} A x_{n}\right),
$$

for every $n=0,1,2, \cdots$, where $A$ is $\alpha$-cocoercive, $x_{0}=x \in C,\left\{\alpha_{n}\right\}$ is a sequence in $(0,1)$, and $\left\{\lambda_{n}\right\}$ is a sequence in $(0,2 \alpha)$. And, the sequence $\left\{x_{n}\right\}$ generated by (1.5) was proved to converge strongly to $\mathrm{q} \in \operatorname{Fix}(\mathrm{S}) \cap \operatorname{VI}(\mathrm{C}, \mathrm{A})$.

In 2007, Yao and Yao [17] extended (1.5) to the following iterative scheme:

$$
\left\{\begin{array}{l}
y_{n}=P_{c}\left(x_{n}-\lambda_{n} A x_{n}\right), \\
x_{n+1}=\alpha_{n} u+\beta_{n} x_{n}+\gamma_{n} S P_{C}\left(y_{n}-\lambda_{n} A y_{n}\right)
\end{array}\right.
$$

where $\left\{\alpha_{n}\right\},\left\{\beta_{n}\right\}$, and $\left\{\gamma_{n}\right\}$ are three sequences in $[0,1]$ and $\left\{\lambda_{n}\right\}$ is a sequence in $[0,2 \alpha]$. And, the sequence $\left\{x_{n}\right\}$ defined by (1.6) was proved to converge strongly to a common element of the set of fixed points of a nonexpansive mapping and the set of solutions of the variational inequality for $\alpha$-inverse-strongly monotone mappings under some parameters controlling conditions.

In 2012, Piri [9] proposed an iteration method for finding an infinite family of nonexpansive mappings, the set of solutions of systems of equilibrium problems and the set of solutions of systems of variational inequalities for two strongly monotone mappings in a real Hilbert space. In 2014, Bnouhachem [2] proposed a modified projection method for computing a common solution of a system of variational inequalities, a split equilibrium problem, and a hierarchical fixed-point problem in Hilbert space, and proved the strong convergence of the iteration sequences. Since the iterative algorithms played an important role for solving integral and differential equations, optimization problems, image reconstruction problems, game theory and other fields such as $[5,16,19,20,22]$, the convergence and construction of the iteration algorithm for computing fixed points has attracted more and more attentions see, e.g., [3, 6, 12, 18, 21].

Motivated by the above related results in this field, a new general iterative process is constructed:

$$
\left\{\begin{array}{l}
F\left(y_{n}, u\right)+\frac{1}{r_{n}}\left\langle u-y_{n}, y_{n}-x_{n}\right\rangle \geqslant 0, \quad \forall u \in H, \\
\left.x_{n+1}=\alpha_{n} \gamma f\left(x_{n}\right)+\beta_{n} x_{n}+\left(\left(1-\beta_{n}\right) I-\alpha_{n} A\right)\right) S P_{C}\left(I-s_{n} B\right) y_{n}, \quad \forall n \geqslant 1,
\end{array}\right.
$$

where $A$ is a linear bounded operator and $B$ is relaxed cocoercive. The strong convergence on the sequence $\left\{x_{n}\right\}$ generated by (1.7) to a common element of the set of fixed points of a nonexpansive mapping, the set of solution of the variational inequalities for a relaxed cocoercive mapping and the set of solutions of the equilibrium problem will be proved, and the common element also solves another variational inequality:

$$
\langle\gamma \mathrm{f}(\mathrm{q})-\mathrm{Aq}, \mathrm{q}-\mathrm{p}\rangle \leqslant 0, \quad \forall \mathrm{p} \in \mathscr{F},
$$

where $\mathscr{F}=\operatorname{Fix}(S) \cap \mathrm{VI}(\mathrm{S}, \mathrm{C}) \cap \mathrm{EP}(\mathrm{F})$ and is also the optimality condition for the minimization problem $\min _{x \in \mathscr{F}} \frac{1}{2}\langle A x, x\rangle-h(x)$, where $h$ is a potential function for $\gamma f$.

\section{Preliminaries}

Let $\mathrm{H}$ be a Hilbert space, whose inner product and norm are denoted by $\langle\cdot\rangle$ and $\|\cdot\|$, respectively. Let $\mathrm{C}$ be a nonempty closed convex subset of $\mathrm{H}$ and let $\mathrm{A}: \mathrm{C} \rightarrow \mathrm{H}$ be a nonlinear map. Let $\mathrm{P}_{\mathrm{C}}$ be the projection of $\mathrm{H}$ onto the convex subset $\mathrm{C}$. The classical variational inequality which is denoted by $\operatorname{VI}(A, C)$ is used to find $u \in C$ such that

$$
\langle A u, v-u\rangle \geqslant 0
$$


for all $v \in \mathrm{C}$. For a given $z \in \mathrm{H}, \mathrm{u} \in \mathrm{C}$ satisfies the inequality

$$
\langle u-z, v-u\rangle \geqslant 0, \quad \forall v \in C,
$$

if and only if $u=P_{c} z$. It is known that projection operator $P_{C}$ is nonexpansive. Furthermore, for $x \in H$ and $u \in C$,

$$
u=P_{c}(x) \Leftrightarrow\left\langle x-P_{c} x, P_{c} x-y\right\rangle \geqslant 0, \forall y \in C .
$$

It is also known that $\mathrm{P}_{\mathrm{C}}$ satisfies

$$
\left\langle x-y, P_{c} x-P_{c} y\right\rangle \geqslant\left\|P_{c} x-P_{c} y\right\|^{2} \text { for all } x, y \in H .
$$

Recall that

(1) B is called $v$-strongly monotone, if for each $x, y \in C$, we have

$$
\langle\mathrm{B} x-\mathrm{B} y, x-y\rangle \geqslant v\|x-y\|^{2},
$$

for a constant $v>0$. This implies that

$$
\|B x-B y\| \geqslant v\|x-y\|,
$$

that is, $B$ is $v$-expansive and when $v=1$, it is expansive.

(2) $B$ is called $\mu$-cocoercive [15], if for each $x, y \in C$, we have $\langle B x-B y, x-y\rangle \geqslant \mu\|B x-B y\|^{2}$, for a constant $\mu>0$. Clearly, every $\mu$-cocoercive map B is $1 / \mu$-Lipschitz continuous.

(3) $B$ is called $-\mu$-cocoercive, if there exists a constant $\mu>0$ such that

$$
\langle\mathrm{B} x-\mathrm{B} y, x-\mathrm{y}\rangle \geqslant(-\mu)\|\mathrm{B} x-\mathrm{B} y\|^{2}, \quad \forall x, y \in \mathrm{C} .
$$

(4) B is said to be relaxed $(\mu, v)$-cocoercive, if there exist two constants $\mu, v>0$ such that

$$
\langle B x-B y, x-y\rangle \geqslant(-\mu)\|B x-B y\|^{2}+v\|x-y\|^{2}, \quad \forall x, y \in C .
$$

For $\mu=0, B$ is $v$-strongly monotone. This class of maps is more general than the class of strongly monotone maps. We can have the following implication: $v$-strongly monotone $\Rightarrow$ relaxed $(\mu, v)$ cocoercivity.

(5) A set-valued mapping $T: H \rightarrow 2^{H}$ is called monotone if for all $x, y \in H, f \in T x$ and $g \in T y$ imply $\langle x-y, f-g\rangle \geqslant 0$. A monotone mapping $T: H \rightarrow 2^{\mathrm{H}}$ is maximal if the graph $G(T)$ of $T$ is not properly contained in the graph of any other monotone mapping. It is known that a monotone mapping $T$ is maximal if and only if for $(x, f) \in H \times H,\langle x-y, f-g\rangle \geqslant 0$ for every $(y, g) \in G(T)$ implies $f \in T x$. Let $B$ be a monotone map of $C$ into $H$ and let $N_{C} v$ be the normal cone to $C$ at $v \in C$, i.e., $N_{C} v=\{w \in H:\langle v-u, w\rangle \geqslant 0, \forall u \in C\}$ and define

$$
\mathrm{T} v= \begin{cases}\mathrm{B} v+\mathrm{N}_{\mathrm{c}} v, & v \in \mathrm{C}, \\ \phi, & v \notin \mathrm{C} .\end{cases}
$$

Then $T$ is the maximal monotone and $0 \in T v$ if and only if $v \in V I(B, C)$; the relative content can be found in [11].

In this paper, for solving the equilibrium problems for a bifunction $F: C \times C \rightarrow R$, the following assumptions on $F$ will be used:

(C1) $F(x, x)=0$ for all $x \in C$; 
(C2) $F$ is monotone, i.e., $F(x, y)+F(y, x) \leqslant 0$ for all $x, y \in C$;

(C3) For each $x, y, z \in C, \lim _{t \downarrow 0} F(t z+(1-t) x, y) \leqslant F(x, y)$;

(C4) For each $x \in C, y \mapsto F(x, y)$ is convex and lower semicontinuous.

If an equilibrium bifunction $\mathrm{F}: \mathrm{C} \times \mathrm{C} \rightarrow \mathrm{R}$ satisfies conditions (C1)-(C4), then we have the following two important results.

Lemma 2.1 ([1]). Let $\mathrm{C}$ be a nonempty closed convex subset of $\mathrm{H}$ and let $\mathrm{F}$ be an equilibrium bifunction of $\mathrm{C} \times \mathrm{C}$ into $\mathrm{R}$ satisfying conditions (C1)-(C4). Let $\mathrm{r}>0$ and $\mathrm{x} \in \mathrm{C}$. Then, there exists $\mathrm{y} \in \mathrm{C}$ such that

$$
\mathrm{F}(\mathrm{y}, z)+\frac{1}{\mathrm{r}}\langle z-\mathrm{y}, \mathrm{y}-\mathrm{x}\rangle \geqslant 0, \text { for all } z \in \mathrm{C} .
$$

Lemma 2.2 ([4]). Assume that $\mathrm{F}$ satisfies the same assumptions as Lemma 2.1. For $\mathrm{r}>0$ and $\mathrm{x} \in \mathrm{C}$, define a mapping $\mathrm{T}_{\mathrm{r}}: \mathrm{H} \rightarrow \mathrm{C}$ as follows:

$$
\mathrm{T}_{\mathrm{r}}(x)=\left\{\mathrm{y} \in \mathrm{C}: \mathrm{F}(\mathrm{y}, z)+\frac{1}{\mathrm{r}}\langle z-y, y-x\rangle \geqslant 0, \forall z \in C\right\},
$$

for all $\mathrm{y} \in \mathrm{H}$. Then, the following items hold:

(1) $\mathrm{T}_{\mathrm{r}}$ is single-valued;

(2) $\mathrm{T}_{\mathrm{r}}$ is firmly nonexpansive, i.e., for any $\mathrm{x}, \mathrm{y} \in \mathrm{H},\left\|\mathrm{T}_{\mathrm{r}} \mathrm{x}-\mathrm{T}_{\mathrm{r}} \mathrm{y}\right\|^{2} \leqslant\left\langle\mathrm{~T}_{\mathrm{r}} x-\mathrm{T}_{\mathrm{r}} \mathrm{y}, \mathrm{x}-\mathrm{y}\right\rangle$;

(3) $\operatorname{Fix}\left(\mathrm{T}_{\mathrm{r}}\right)=\mathrm{EP}(\mathrm{F})$;

(4) $\mathrm{EP}(\mathrm{F})$ is closed and convex.

We also need the following lemmas for proving our main results.

Lemma 2.3 ([13]). Let $\left\{x_{n}\right\}$ and $\left\{y_{n}\right\}$ be bounded sequences in a Banach space $X$ and let $\left\{\beta_{n}\right\}$ be a sequence in $[0,1]$ with $0<\liminf _{n \rightarrow \infty} \beta_{n} \leqslant \limsup _{n \rightarrow \infty} \beta_{n}<1$. Suppose $x_{n+1}=\left(1-\beta_{n}\right) y_{n}+\beta_{n} x_{n}$ for all integers $n \geqslant 0$, and $\limsup _{n \rightarrow \infty}\left(\left\|y_{n+1}-y_{n}\right\|-\left\|x_{n+1}-x_{n}\right\|\right) \leqslant 0$. Then, $\lim _{n \rightarrow \infty}\left\|y_{n}-x_{n}\right\|=0$.

Lemma 2.4 ([8]). Assume $\left\{a_{n}\right\}$ is a sequence of nonnegative real numbers such that

$$
a_{n+1} \leqslant\left(1-\gamma_{n}\right) a_{n}+\delta_{n}
$$

where $\left\{\gamma_{n}\right\}$ is a sequence in $(0,1)$ and $\left\{\delta_{n}\right\}$ is a sequence such that

(1) $\sum_{n=1}^{\infty} \gamma_{n}=\infty$;

(2) $\limsup _{n \rightarrow \infty} \frac{\delta_{n}}{\gamma_{n}} \leqslant 0$ or $\sum_{n=1}^{\infty}\left|\delta_{n}\right|<\infty$.

Then $\lim _{n \rightarrow \infty} a_{n}=0$.

\section{Main results}

Theorem 3.1. Let $\mathrm{C}$ be a nonempty closed convex subset of a Hilbert space $\mathrm{H}$. Let $\mathrm{F}: \mathrm{C} \times \mathrm{C} \rightarrow \mathfrak{R}$ be a bifunction which satisfies (C1)-(C4), $\mathrm{S}$ be a nonexpansive mapping of $\mathrm{C}$ into $\mathrm{H}$ and $\mathrm{B}$ be a $\lambda$-Lipschitzian, relaxed $(\mu, v)$ cocoercive map of $\mathrm{C}$ into $\mathrm{H}$ such that $\mathscr{F}=\operatorname{Fix}(\mathrm{S}) \cap \mathrm{EP}(\mathrm{F}) \cap \mathrm{VI}(\mathrm{B}, \mathrm{C}) \neq \phi$. Let $\mathrm{A}$ be a strongly positive linear bounded operator with coefficient $\bar{\gamma}>0$, and assume that $0<\gamma<\frac{\bar{\gamma}}{\alpha}$. Let $\mathrm{f}$ be a contraction of $\mathrm{H}$ into itself with a coefficient $\alpha \in(0,1),\left\{x_{n}\right\}$ and $\left\{y_{n}\right\}$ be sequences generated by $(1.7)$ with $x_{1} \in H$, where $\left\{\alpha_{n}\right\} \subset[0,1]$ and $\left\{r_{n}\right\},\left\{s_{n}\right\} \subset[0, \infty)$ satisfy 
(i) $\lim _{n \rightarrow \infty} \alpha_{n}=0$ and $\sum_{n=1}^{\infty} \alpha_{n}=\infty$;

(ii) $0<\liminf _{n \rightarrow \infty} \beta_{n} \leqslant \limsup _{n \rightarrow \infty} \beta_{n}<1$;

(iii) $\liminf _{n \rightarrow \infty} r_{n}>0, \lim _{n \rightarrow \infty}\left(r_{n+1}-r_{n}\right)=0$, and $\sum_{n=1}^{\infty}\left|s_{n+1}-s_{n}\right|<\infty$.

(iv) $\left\{s_{n}\right\} \subset[a, b]$ for some $a, b$ with $0 \leqslant a \leqslant b \leqslant \frac{2\left(v-\mu \lambda^{2}\right)}{\lambda^{2}}$.

Then, the sequences $\left\{x_{n}\right\}$ and $\left\{y_{n}\right\}$ converge strongly to $q \in \mathscr{F}$, where $q=P_{\mathscr{F}}(\gamma f+(I-A))(q)$ is a unique solution of the following variational inequality

$$
\langle\gamma \mathrm{f}(\mathrm{q})-\mathrm{Aq}, \mathrm{p}-\mathrm{q}\rangle \leqslant 0, \quad \forall \mathrm{p} \in \mathscr{F},
$$

which is the optimality condition for the minimization problem

$$
\min _{x \in \mathscr{F}} \frac{1}{2}\langle A x, x\rangle-h(x)
$$

where $\mathrm{h}$ is a potential function for $\gamma \mathrm{f}$.

Proof. For the control conditions (i) and (ii), we may assume, without loss of generality, that $\alpha_{n} \leqslant(1-$ $\left.\beta_{n}\right)\|A\|^{-1}$. Since $A$ is linear bounded self-adjoint operator on $H$, then

$$
\|A\|=\sup \{|\langle A u, u\rangle|: u \in H,\|u\|=1\},
$$

Observe that

$$
\left\langle\left(\left(1-\beta_{n}\right) I-\alpha_{n} A\right) u, u\right\rangle=1-\beta_{n}-\alpha_{n}\langle A u, u\rangle \geqslant 1-\beta_{n}-\alpha_{n}\|A\| \geqslant 0,
$$

that is to say $\left(1-\beta_{n}\right) I-\alpha_{n} A$ is positive. It follows that

$$
\begin{aligned}
\left\|\left(1-\beta_{n}\right) I-\alpha_{n} A\right\| & =\sup \left\{\left\langle\left(\left(1-\beta_{n}\right) I-\alpha_{n} A\right) u, u\right\rangle: u \in H,\|u\|=1\right\} \\
& =\sup \left\{1-\beta_{n}-\alpha_{n}\langle A u, u\rangle: u \in H,\|u\|=1\right\} \\
& \leqslant 1-\beta_{n}-\alpha_{n} \bar{\gamma} .
\end{aligned}
$$

Step 1. We show that $I-s_{n} B$ is nonexpansive. Indeed, from the relaxed $(\mu, v)$-cocoercive and $\lambda$-Lipschitzian definition on $B$ and condition (iv), we have

$$
\begin{aligned}
\left\|\left(I-s_{n} B\right) x-\left(I-s_{n} B\right) y\right\|^{2} & =\left\|(x-y)-s_{n}(B x-B y)\right\|^{2} \\
& =\|x-y\|^{2}-2 s_{n}\langle x-y, B x-B y\rangle+s_{n}^{2} \mid B x-B \|^{2} \\
& \leqslant\|x-y\|^{2}-2 s_{n}\left[-\mu\|B x-B y\|^{2}+v\|x-y\|^{2}\right]+s_{n}^{2}\|B x-B y\|^{2} \\
& \leqslant\|x-y\|^{2}+2 s_{n} \lambda^{2} \mu\|x-y\|^{2}-2 s_{n} v\|x-y\|^{2}+\lambda^{2} s_{n}^{2}\|x-y\|^{2} \\
& =\left(1+2 s_{n} \lambda^{2} \mu-2 s_{n} v+\lambda^{2} s_{n}^{2}\right)\|x-y\|^{2} \\
& \leqslant\|x-y\|^{2},
\end{aligned}
$$

which implies that the mapping $\mathrm{I}-\mathrm{s}_{\mathrm{n}} \mathrm{B}$ is nonexpansive.

Step 2. We show $\left\{x_{n}\right\}$ is bounded. Picking $p \in \mathscr{F}$, by the definition of $T_{r}$, and noting that $y_{n}=T_{r_{n}} x_{n}$, we have that

$$
\left\|y_{n}-p\right\|=\left\|T_{r_{n}}\right\| x_{n}-T_{r_{n}} p \leqslant\left\|x_{n}-p\right\| .
$$

Set $\rho_{n}=P_{C}\left(I-s_{n} B\right) y_{n}$, since $p \in V I(B, C)$, we have $p=P_{C}\left(I-s_{n} B\right) p$. Therefore, we obtain

$$
\begin{aligned}
\left\|\rho_{n}-p\right\| & =\left\|P_{C}\left(I-s_{n} B\right) y_{n}-P_{C}\left(I-s_{n} B\right) p\right\| \\
& \leqslant\left\|\left(I-s_{n} B\right) y_{n}-\left(I-s_{n} B\right) p\right\| \\
& \leqslant\left\|y_{n}-p\right\| \leqslant\left\|x_{n}-p\right\| .
\end{aligned}
$$


Using (3.1), we have

$$
\begin{aligned}
\left\|x_{n+1}-p\right\| & =\left\|\alpha_{n}\left(\gamma f\left(x_{n}\right)-A p\right)+\beta_{n}\left(x_{n}-p\right)+\left(\left(1-\beta_{n}\right) I-\alpha_{n} A\right)\left(S P_{C}\left(I-s_{n} B\right) y_{n}-p\right)\right\| \\
& \leqslant\left(1-\beta_{n}-\alpha_{n} \bar{\gamma}\right)\left\|\left(I-s_{n} B\right) y_{n}-p\right\|+\beta_{n}\left\|x_{n}-p\right\|+\alpha_{n}\left\|\gamma f\left(x_{n}\right)-A p\right\| \\
& \leqslant\left(1-\beta_{n}-\alpha_{n} \bar{\gamma}\right)\left\|y_{n}-p\right\|+\beta_{n}\left\|x_{n}-p\right\|+\alpha_{n} \gamma\left\|f\left(x_{n}\right)-f(p)\right\|+\alpha_{n}\|\gamma f(p)-A p\| \\
& \leqslant\left(1-\alpha_{n} \bar{\gamma}\right)\left\|x_{n}-p\right\|+\alpha_{n} \gamma \alpha\left\|x_{n}-p\right\|+\alpha_{n}\|\gamma f(p)-A p\| \\
& \leqslant\left(1-(\bar{\gamma}-\gamma \alpha) \alpha_{n}\right)\left\|x_{n}-p\right\|+\alpha_{n}\|\gamma f(p)-A p\| .
\end{aligned}
$$

It follows from (3.2) and induction, we can get

$$
\left\|x_{n}-p\right\| \leqslant \max \left\{\left\|x_{0}-p\right\|, \frac{\|\gamma f(p)-A p\|}{\bar{\gamma}-\gamma \alpha}\right\}, \quad n \geqslant 0 .
$$

Therefore, $\left\{x_{n}\right\}$ is bounded. We also obtain that $\left\{y_{n}\right\},\left\{f\left(x_{n}\right)\right\}$, and $\left\{\rho_{n}\right\}$ are all bounded.

Step 3. We show that

$$
\lim _{n \rightarrow \infty}\left\|x_{n+1}-x_{n}\right\|=0 .
$$

Observing that $y_{n}=T_{r_{n}} x_{n}$ and $y_{n+1}=T_{r_{n+1}} x_{n+1}$, we have

$$
F\left(y_{n}, u\right)+\frac{1}{r_{n}}\left\langle u-y_{n}, y_{n}-x_{n}\right\rangle \geqslant 0 \text { for all } u \in C \text {. }
$$

and

$$
F\left(y_{n+1}, u\right)+\frac{1}{r_{n+1}}\left\langle u-y_{n+1}, y_{n+1}-x_{n+1}\right\rangle \geqslant 0 \text { for all } u \in C .
$$

Putting $u=y_{n+1}$ in (3.3) and $u=y_{n}$ in (3.4), we get

$$
F\left(y_{n}, y_{n+1}\right)+\frac{1}{r_{n}}\left\langle y_{n+1}-y_{n}, y_{n}-x_{n}\right\rangle \geqslant 0
$$

and

$$
F\left(y_{n+1}, y_{n}\right)+\frac{1}{r_{n+1}}\left\langle y_{n}-y_{n+1}, y_{n+1}-x_{n+1}\right\rangle \geqslant 0
$$

It follows from (C2) that

$$
\left\langle y_{n+1}-y_{n}, \frac{y_{n}-x_{n}}{r_{n}}-\frac{y_{n+1}-x_{n+1}}{r_{n+1}}\right\rangle \geqslant 0 .
$$

So we can get,

$$
\left\langle y_{n+1}-y_{n}, y_{n}-y_{n+1}+y_{n+1}-x_{n}-\frac{r_{n}}{r_{n+1}}\left(y_{n+1}-x_{n+1}\right)\right\rangle \geqslant 0 .
$$

Without loss of generality, we assume that there exists a real number $m$ such that $r_{n}>m>0$ for all $n$, it follows that

$$
\left\|y_{n+1}-y_{n}\right\|^{2} \leqslant\left\|y_{n+1}-y_{n}\right\|\left(\left\|x_{n+1}-x_{n}\right\|+\left|1-\frac{r_{n}}{r_{n+1}}\right|\left\|y_{n+1}-x_{n+1}\right\|\right)
$$

i.e.,

$$
\begin{aligned}
\left\|y_{n+1}-y_{n}\right\| & \leqslant\left\|x_{n+1}-x_{n}\right\|+\left|1-\frac{r_{n}}{r_{n+1}}\right| \mid y_{n+1}-x_{n+1} \| \\
& \leqslant\left\|x_{n+1}-x_{n}\right\|+\frac{M}{m}\left|r_{n+1}-r_{n}\right|,
\end{aligned}
$$

where $M$ is an appropriate constant such that $M \geqslant \sup _{n \geqslant 1}\left\|y_{n}-x_{n}\right\|$. Note that

$$
\begin{aligned}
\left\|\rho_{n+1}-\rho_{n}\right\| & =\left\|P_{C}\left(I-s_{n+1} B\right) y_{n+1}-P_{C}\left(I-s_{n} B\right) y_{n}\right\| \\
& \leqslant\left\|\left(I-s_{n+1} B\right) y_{n+1}-\left(I-s_{n} B\right) y_{n}\right\| \\
& =\left\|\left(I-s_{n+1} B\right) y_{n+1}-\left(I-s_{n+1} B\right) y_{n}+\left(s_{n}-s_{n+1}\right) B y_{n}\right\| \\
& \leqslant\left\|y_{n+1}-y_{n}\right\|+\left|s_{n}-s_{n+1}\right|\left\|B y_{n}\right\| .
\end{aligned}
$$


Substituting (3.5) into (3.6) yields that

$$
\left\|\rho_{n+1}-\rho_{n}\right\| \leqslant\left\|x_{n+1}-x_{n}\right\|+M_{1}\left(\left|r_{n+1}-r_{n}\right|+\left|s_{n}-s_{n+1}\right|\right),
$$

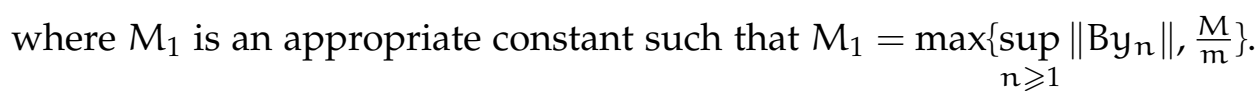

We set $x_{n+1}=\beta_{n} x_{n}+\left(1-\beta_{n}\right) z_{n}$ for all $n \geqslant 0$. From the definition of $z_{n}$, we obtain

$$
\begin{aligned}
z_{n+1}-z_{n} & =\frac{x_{n+2}-\beta_{n+1} x_{n+1}}{1-\beta_{n+1}}-\frac{x_{n+1}-\beta_{n} x_{n}}{1-\beta_{n}} \\
& =\frac{\alpha_{n+1} \gamma f\left(x_{n+1}\right)+\left(\left(1-\beta_{n+1}\right) I-\alpha_{n+1} A\right) S \rho_{n+1}}{1-\beta_{n+1}}-\frac{\alpha_{n} \gamma f\left(x_{n}\right)+\left(\left(1-\beta_{n}\right) I-\alpha_{n} A\right) S \rho_{n}}{1-\beta_{n}} \\
& =\frac{\alpha_{n+1}}{1-\beta_{n+1}}\left(\gamma f\left(x_{n+1}\right)-A S \rho_{n+1}\right)+\frac{\alpha_{n}}{1-\beta_{n}}\left(\gamma f\left(x_{n}\right)-A S \rho_{n}\right)+S \rho_{n+1}-S \rho_{n} .
\end{aligned}
$$

By using (3.7), it follows that

$$
\begin{aligned}
\left\|z_{n+1}-z_{n}\right\|-\left\|x_{n+1}-x_{n}\right\| \leqslant & \frac{\alpha_{n+1}}{1-\beta_{n+1}}\left(\left\|\gamma f\left(x_{n+1}\right)\right\|+\left\|A S \rho_{n+1}\right\|\right)+\frac{\alpha_{n}}{1-\beta_{n}}\left(\left\|\gamma f\left(x_{n}\right)\right\|+\left\|A S \rho_{n}\right\|\right) \\
& +\left\|\rho_{n+1}-\rho_{n}\right\|-\left\|x_{n+1}-x_{n}\right\| \\
\leqslant & \frac{\alpha_{n+1}}{1-\beta_{n+1}}\left(\left\|\gamma f\left(x_{n+1}\right)\right\|+\left\|A S \rho_{n+1}\right\|\right)+\frac{\alpha_{n}}{1-\beta_{n}}\left(\left\|\gamma f\left(x_{n}\right)\right\|+\left\|A S \rho_{n}\right\|\right) \\
& +\left\|x_{n+1}-x_{n}\right\|+M_{1}\left(\left|r_{n+1}-r_{n}\right|+\left|s_{n+1}-s_{n}\right|\right)-\left\|x_{n+1}-x_{n}\right\| \\
= & \frac{\alpha_{n+1}}{1-\beta_{n+1}}\left(\left\|\gamma f\left(x_{n+1}\right)\right\|+\left\|A S \rho_{n+1}\right\|\right)+\frac{\alpha_{n}}{1-\beta_{n}}\left(\left\|\gamma f\left(x_{n}\right)\right\|+\left\|A S \rho_{n}\right\|\right) \\
& +M_{1}\left(\left|r_{n+1}-r_{n}\right|+\left|s_{n+1}-s_{n}\right|\right) .
\end{aligned}
$$

From the conditions (i) and (iii), the last inequality implies that

$$
\lim _{n \rightarrow \infty}\left(\left\|z_{n+1}-z_{n}\right\|-\left\|x_{n+1}-x_{n}\right\|\right) \leqslant 0
$$

Hence by Lemma 2.3, we have $\lim _{n \rightarrow \infty}\left\|z_{n}-x_{n}\right\|=0$. Consequently,

$$
\lim _{n \rightarrow \infty}\left\|x_{n+1}-x_{n}\right\|=\lim _{n \rightarrow \infty}\left(1-\beta_{n}\right)\left\|z_{n}-x_{n}\right\|=0 .
$$

Step 4. We show that $\left\|x_{n}-y_{n}\right\| \rightarrow 0$. Since $x_{n+1}=\alpha_{n} \gamma f\left(x_{n}\right)+\beta_{n} x_{n}+\left(\left(1-\beta_{n}\right) I-\alpha_{n} A\right) S \rho_{n}$, we have

$$
\left\|x_{n}-S \rho_{n}\right\| \leqslant\left\|x_{n}-x_{n+1}\right\|+\left\|x_{n+1}-S \rho_{n}\right\| \leqslant\left\|x_{n}-x_{n+1}\right\|+\alpha_{n}\left\|\gamma f\left(x_{n}\right)-A S \rho_{n}\right\|+\beta_{n}\left\|x_{n}-S \rho_{n}\right\| .
$$

So, we get

$$
\left\|x_{n}-S \rho_{n}\right\| \leqslant \frac{1}{1-\beta_{n}}\left\|x_{n}-x_{n+1}\right\|+\frac{\alpha_{n}}{1-\beta_{n}}\left\|\gamma f\left(x_{n}\right)-A S \rho_{n}\right\|
$$

By condition (i) and using (3.8), we obtain

$$
\lim _{n \rightarrow \infty}\left\|x_{n}-S \rho_{n}\right\|=0 .
$$

For $\mathrm{p} \in \mathscr{F}$, note that $\mathrm{T}_{\mathrm{r}}$ is firmly nonexpansive, then we have

$$
\begin{aligned}
\left\|y_{n}-p\right\|^{2}=\left\|T_{r_{n}} x_{n}-T_{r_{n}} p\right\|^{2} \leqslant\left\langle T_{r_{n}} x_{n}-T_{r_{n}} p, x_{n}-p\right\rangle & =\left\langle y_{n}-p, x_{n}-p\right\rangle \\
& =\frac{1}{2}\left(\left\|y_{n}-p\right\|^{2}+\left\|x_{n}-p\right\|^{2}-\left\|x_{n}-y_{n}\right\|^{2}\right),
\end{aligned}
$$

and hence $\left\|y_{n}-p\right\|^{2} \leqslant\left\|x_{n}-p\right\|^{2}-\left\|x_{n}-p\right\|^{2}$. 
Therefore, we have

$$
\begin{aligned}
l c l\left\|x_{n+1}-p\right\|^{2}= & \left\|\alpha_{n}\left(\gamma f\left(x_{n}\right)-A p\right)+\beta_{n}\left(x_{n}-S \rho_{n}\right)+\left(I-\alpha_{n} A\right)\left(S \rho_{n}-p\right)\right\|^{2} \\
\leqslant & \left\|\left(I-\alpha_{n} A\right)\left(S \rho_{n}-p\right)+\beta_{n}\left(x_{n}-S \rho_{n}\right)\right\|^{2}+2 \alpha_{n}\left\langle\gamma f\left(x_{n}\right)-A p, x_{n+1}-p\right\rangle \\
\leqslant & {\left[\left\|\left(I-\alpha_{n} A\right)\left(S \rho_{n}-p\right)\right\|+\left\|\beta_{n}\left(x_{n}-S \rho_{n}\right)\right\|\right]^{2}+2 \alpha_{n}\left\|\gamma f\left(x_{n}\right)-A p\right\|\left\|x_{n+1}-p\right\| } \\
\leqslant & {\left[\left(1-\alpha_{n} \bar{\gamma}\right)\left\|\rho_{n}-p\right\|+\beta_{n}\left\|x_{n}-S \rho_{n}\right\|\right]^{2}+2 \alpha_{n}\left\|\gamma f\left(x_{n}\right)-A p\right\|\left\|x_{n+1}-p\right\| } \\
= & \left(1-\alpha_{n} \bar{\gamma}\right)^{2}\left\|y_{n}-p\right\|^{2}+\beta_{n}^{2}\left\|x_{n}-S \rho_{n}\right\|^{2}+2\left(1-\alpha_{n} \bar{\gamma}\right) \beta_{n}\left\|\rho_{n}-p\right\|\left\|x_{n}-S \rho_{n}\right\| \\
& +2 \alpha_{n}\left\|\gamma f\left(x_{n}\right)-A p\right\|\left\|x_{n+1}-p\right\| \\
\leqslant & \left(1-\alpha_{n} \bar{\gamma}\right)^{2}\left(\left\|x_{n}-p\right\|^{2}-\left\|x_{n}-y_{n}\right\|^{2}\right)+\beta_{n}^{2}\left\|x_{n}-S \rho_{n}\right\|^{2} \\
& +2\left(1-\alpha_{n} \bar{\gamma}\right) \beta_{n}\left\|\rho_{n}-p\right\|\left\|x_{n}-S \rho_{n}\right\|+2 \alpha_{n}\left\|\gamma f\left(x_{n}\right)-A p\right\|\left\|x_{n+1}-p\right\| \\
= & \left(1-2 \alpha_{n} \bar{\gamma}+\left(\alpha_{n} \bar{\gamma}\right)^{2}\right)\left\|x_{n}-p\right\|^{2}-\left(1-\alpha_{n} \bar{\gamma}\right)^{2}\left\|x_{n}-y_{n}\right\|^{2}+\beta_{n}^{2}\left\|x_{n}-S \rho_{n}\right\|^{2} \\
& +2\left(1-\alpha_{n} \bar{\gamma}\right) \beta_{n}\left\|\rho_{n}-p\right\|\left\|x_{n}-S \rho_{n}\right\|+2 \alpha_{n}\left\|\gamma f\left(x_{n}\right)-A p\right\|\left\|x_{n+1}-p\right\| \\
\leqslant & \left\|x_{n}-p\right\|^{2}+\alpha_{n} \bar{\gamma}^{2}\left\|x_{n}-p\right\|^{2}-\left(1-\alpha_{n} \bar{\gamma}\right)^{2}\left\|x_{n}-y_{n}\right\|^{2}+\beta_{n}^{2}\left\|x_{n}-S \rho_{n}\right\|^{2} \\
& +2\left(1-\alpha_{n} \bar{\gamma}\right) \beta_{n}\left\|\rho_{n}-p\right\|\left\|x_{n}-S \rho_{n}\right\|+2 \alpha_{n}\left\|\gamma f\left(x_{n}\right)-A p\right\|\left\|x_{n+1}-p\right\| .
\end{aligned}
$$

Then, we have

$$
\begin{aligned}
\left(1-\alpha_{n} \bar{\gamma}\right)^{2}\left\|x_{n}-y_{n}\right\|^{2} \leqslant & \left\|x_{n}-p\right\|^{2}-\left\|x_{n+1}-p\right\|^{2}+\alpha_{n} \bar{\gamma}^{2}\left\|x_{n}-p\right\|^{2}+\beta_{n}^{2}\left\|x_{n}-S \rho_{n}\right\|^{2} \\
& +2\left(1-\alpha_{n} \bar{\gamma}\right) \beta_{n}\left\|\rho_{n}-p\right\|\left\|x_{n}-S \rho_{n}\right\|+2 \alpha_{n}\left\|\gamma f\left(x_{n}\right)-A p\right\|\left\|x_{n+1}-p\right\| \\
\leqslant & \left(\left\|x_{n}-p\right\|+\left\|x_{n+1}-p\right\|\right) \times\left\|x_{n+1}-x_{n}\right\|+\alpha_{n} \bar{\gamma}^{2}\left\|x_{n}-p\right\|^{2} \\
& +\beta_{n}^{2}\left\|x_{n}-S \rho_{n}\right\|^{2}+2 \alpha_{n}\left\|\gamma f\left(x_{n}\right)-A p\right\|\left\|x_{n+1}-p\right\| \\
& +2\left(1-\alpha_{n} \bar{\gamma}\right) \beta_{n}\left\|\rho_{n}-p\right\|\left\|x_{n}-S \rho_{n}\right\| .
\end{aligned}
$$

From (3.8)-(3.10) and condition (i), we have

$$
\lim _{n \rightarrow \infty}\left\|x_{n}-y_{n}\right\|=0 \text {. }
$$

Step 5. We will show that $\mathrm{P}_{\mathscr{F}}(\gamma f+(I-A))$ has a unique fixed point. For $p \in \mathscr{F}$, we have

$$
\begin{aligned}
\left\|\rho_{n}-p\right\|^{2} & =\left\|P_{C}\left(I-s_{n} B\right) y_{n}-P_{C}\left(I-s_{n} B\right) p\right\|^{2} \\
& \leqslant\left\|\left(y_{n}-p\right)-s_{n}\left(B y_{n}-B p\right)\right\|^{2} \\
& =\left\|y_{n}-p\right\|^{2}-2 s_{n}\left\langle y_{n}-p, B y_{n}-B p\right\rangle+s_{n}^{2}\left\|B y_{n}-B p\right\|^{2} \\
& \leqslant\left\|x_{n}-p\right\|^{2}-2 s_{n}\left[-\mu\left\|B y_{n}-B p\right\|^{2}+v\left\|y_{n}-p\right\|^{2}\right]+s_{n}^{2}\left\|B y_{n}-B p\right\|^{2} \\
& \leqslant\left\|x_{n}-p\right\|^{2}+2 s_{n} \mu\left\|B y_{n}-B p\right\|^{2}-2 s_{n} v\left\|y_{n}-p\right\|^{2}+s_{n}^{2}\left\|B y_{n}-B p\right\|^{2} \\
& \leqslant\left\|x_{n}-p\right\|^{2}+\left(2 s_{n} \mu+s_{n}^{2}-\frac{2 s_{n} v}{\lambda^{2}}\right)\left\|B y_{n}-B p\right\|^{2} .
\end{aligned}
$$

Observe that

$$
\begin{aligned}
\left\|x_{n+1}-p\right\|^{2}= & \left\|\alpha_{n}\left(\gamma f\left(x_{n}\right)-A p\right)+\beta_{n}\left(x_{n}-S \rho_{n}\right)+\left(I-\alpha_{n} A\right)\left(S \rho_{n}-p\right)\right\|^{2} \\
\leqslant & \left\|\left(I-\alpha_{n} A\right)\left(S \rho_{n}-p\right)+\beta_{n}\left(x_{n}-S \rho_{n}\right)\right\|^{2}+2 \alpha_{n}\left\langle\gamma f\left(x_{n}\right)-A p, x_{n+1}-p\right\rangle \\
\leqslant & {\left[\left\|\left(I-\alpha_{n} A\right)\left(S \rho_{n}-p\right)\right\|+\left\|\beta_{n}\left(x_{n}-S \rho_{n}\right)\right\|\right]^{2}+2 \alpha_{n}\left\|\gamma f\left(x_{n}\right)-A p\right\|\left\|x_{n+1}-p\right\| } \\
\leqslant & {\left[\left(1-\alpha_{n} \bar{\gamma}\right)\left\|\rho_{n}-p\right\|+\beta_{n}\left\|x_{n}-S \rho_{n}\right\|\right]^{2}+2 \alpha_{n} \mid \gamma f\left(x_{n}\right)-A p\|\| x_{n+1}-p \| } \\
= & \left(1-\alpha_{n} \bar{\gamma}\right)^{2}\left\|\rho_{n}-p\right\|^{2}+\beta_{n}^{2}\left\|x_{n}-S \rho_{n}\right\|^{2}+2\left(1-\alpha_{n} \bar{\gamma}\right) \beta_{n}\left\|\rho_{n}-p\right\|\left\|x_{n}-S \rho_{n}\right\| \\
& +2 \alpha_{n}\left\|\gamma f\left(x_{n}\right)-A p\right\|\left\|x_{n+1}-p\right\| \\
\leqslant & \left\|\rho_{n}-p\right\|^{2}+\beta_{n}^{2}\left\|x_{n}-S \rho_{n}\right\|^{2}+2 \beta_{n}\left\|\rho_{n}-p\right\|\left\|x_{n}-S \rho_{n}\right\| \\
& +2 \alpha_{n}\left\|\gamma f\left(x_{n}\right)-A p\right\|\left\|x_{n+1}-p\right\| .
\end{aligned}
$$


Substituting (3.11) into (3.12), we can get

$$
\begin{aligned}
\left\|x_{n+1}-p\right\|^{2} \leqslant & \left\|x_{n}-p\right\|^{2}+\left(2 s_{n} \mu+s_{n}^{2}-\frac{2 s_{n} v}{\lambda^{2}}\right)\left\|B y_{n}-B p\right\|^{2}+\beta_{n}^{2}\left\|x_{n}-S \rho_{n}\right\|^{2} \\
& +2 \beta_{n}\left\|\rho_{n}-p\right\|\left\|x_{n}-S \rho_{n}\right\|+2 \alpha_{n}\left\|\gamma f\left(x_{n}\right)-A p\right\|\left\|x_{n+1}-p\right\| .
\end{aligned}
$$

From condition (iv), we can get

$$
\begin{aligned}
\left(\frac{2 a v}{\lambda^{2}}-2 b \mu-b^{2}\right)\left\|B y_{n}-B p\right\|^{2} \leqslant & \left\|x_{n}-p\right\|^{2}-\left\|x_{n+1}-p\right\|^{2}+\beta_{n}^{2}\left\|x_{n}-S \rho_{n}\right\|^{2} \\
& +2 \beta_{n}\left\|\rho_{n}-p\right\|\left\|x_{n}-S \rho_{n}\right\|+2 \alpha_{n}\left\|\gamma f\left(x_{n}\right)-A p\right\|\left\|x_{n+1}-p\right\| \\
\leqslant & \left(\left\|x_{n}-p\right\|+\left\|x_{n+1}-p\right\|\right)\left\|x_{n}-x_{n+1}\right\|+\beta_{n}^{2}\left\|x_{n}-S \rho_{n}\right\|^{2} \\
& +2 \beta_{n}\left\|\rho_{n}-p\right\|\left\|x_{n}-S \rho_{n}\right\|+2 \alpha_{n}\left\|\gamma f\left(x_{n}\right)-A p\right\|\left\|x_{n+1}-p\right\| .
\end{aligned}
$$

From (3.8), (3.9), and condition (i), we have

$$
\lim _{n \rightarrow \infty}\left\|B y_{n}-B p\right\|=0
$$

On the other hand, we have

$$
\begin{aligned}
\left\|\rho_{n}-p\right\|^{2}= & \left\|P_{C}\left(I-s_{n} B\right) y_{n}-P_{C}\left(I-s_{n} B\right) p\right\|^{2} \\
\leqslant & \left\langle\left(I-s_{n} B\right) y_{n}-\left(I-s_{n} B\right) p, \rho_{n}-p\right\rangle \\
= & \frac{1}{2}\left[\left\|\left(I-s_{n} B\right) y_{n}-\left(I-s_{n} B\right) p\right\|^{2}+\left\|\rho_{n}-p\right\|^{2}-\left\|\left(I-s_{n} B\right) y_{n}-\left(I-s_{n} B\right) p-\left(\rho_{n}-p\right)\right\|^{2}\right] \\
\leqslant & \frac{1}{2}\left[\left\|y_{n}-p\right\|^{2}+\left\|\rho_{n}-p\right\|^{2}-\left\|\left(y_{n}-\rho_{n}\right)-s_{n}\left(B y_{n}-B p\right)\right\|^{2}\right] \\
= & \frac{1}{2}\left[\left\|y_{n}-p\right\|^{2}+\left\|\rho_{n}-p\right\|^{2}-\left\|y_{n}-\rho_{n}\right\|^{2}-s_{n}^{2}\left\|B y_{n}-B p\right\|^{2}\right. \\
& \left.+2 s_{n}\left\langle y_{n}-\rho_{n}, B y_{n}-B p\right\rangle\right]
\end{aligned}
$$

which yields that

$$
\left\|\rho_{n}-p\right\|^{2} \leqslant\left\|x_{n}-p\right\|^{2}-\left\|y_{n}-\rho_{n}\right\|^{2}+2 s_{n}\left\|y_{n}-\rho_{n}\right\|\left\|B y_{n}-B p\right\| .
$$

Submitting (3.14) into (3.12) yields that

$$
\begin{aligned}
\left\|x_{n+1}-p\right\|^{2} \leqslant & \left\|x_{n}-p\right\|^{2}-\left\|y_{n}-\rho_{n}\right\|^{2}+2 s_{n}\left\|y_{n}-\rho_{n}\right\|\left\|B y_{n}-B p\right\|+\beta_{n}^{2}\left\|x_{n}-S \rho_{n}\right\|^{2} \\
& +2 \beta_{n}\left\|\rho_{n}-p\right\|\left\|x_{n}-S \rho_{n}\right\|+2 \alpha_{n}\left\|\gamma f\left(x_{n}\right)-A p\right\|\left\|x_{n+1}-p\right\| .
\end{aligned}
$$

It follows that

$$
\begin{aligned}
\left\|y_{n}-\rho_{n}\right\|^{2} \leqslant & \left\|x_{n}-p\right\|^{2}-\left\|x_{n+1}-p\right\|^{2}+2 s_{n}\left\|y_{n}-\rho_{n}\right\|\left\|B y_{n}-B p\right\|+\beta_{n}^{2}\left\|x_{n}-S \rho_{n}\right\|^{2} \\
& +2 \beta_{n}\left\|\rho_{n}-p\right\|\left\|x_{n}-S \rho_{n}\right\|+2 \alpha_{n}\left\|\gamma f\left(x_{n}\right)-A p\right\|\left\|x_{n+1}-p\right\| \\
\leqslant & \left(\left\|x_{n}-p\right\|+\left\|x_{n+1}-p\right\|\right)\left\|x_{n}-x_{n+1}\right\|+2 s_{n}\left\|y_{n}-\rho_{n}\right\|\left\|B y_{n}-B p\right\| \\
& +\beta_{n}^{2}\left\|x_{n}-S \rho_{n}\right\|^{2}+2 \beta_{n}\left\|\rho_{n}-p\right\|\left\|x_{n}-S \rho_{n}\right\|+2 \alpha_{n}\left\|\gamma f\left(x_{n}\right)-A p\right\|\left\|x_{n+1}-p\right\| .
\end{aligned}
$$

From condition (i), (3.8), (3.9), and (3.13), we have that

$$
\lim _{n \rightarrow \infty}\left\|y_{n}-\rho_{n}\right\|=0 .
$$

Then, we can get

$$
\begin{aligned}
\left\|y_{n}-S y_{n}\right\| & \leqslant\left\|S y_{n}-S \rho_{n}\right\|+\left\|S \rho_{n}-x_{n}\right\|+\left\|x_{n}-y_{n}\right\|+\left\|y_{n}-\rho_{n}\right\| \\
& \leqslant 2\left\|y_{n}-\rho_{n}\right\|+\left\|S \rho_{n}-x_{n}\right\|+\left\|x_{n}-y_{n}\right\| .
\end{aligned}
$$


From (3.9), (3.10), and (3.15), we have

$$
\lim _{n \rightarrow \infty}\left\|y_{n}-S y_{n}\right\|=0 \text {. }
$$

Observe that $P_{\mathscr{F}}(\gamma f+(I-A))$ is a contraction. Indeed, for all $x, y \in H$, we have

$$
\begin{aligned}
\left\|P_{\mathscr{F}}(\gamma f+(I-A))(x)-P_{\mathscr{F}}(\gamma f+(I-A))(y)\right\| & \leqslant\|(\gamma f+(I-A))(x)-(\gamma f+(I-A))(y)\| \\
& \leqslant \gamma\|f(x)-f(y)\|+\|I-A\|\|x-y\| \\
& \leqslant \gamma \alpha\|x-y\|+(1-\bar{\gamma})\|x-y\| \\
& <\|x-y\| .
\end{aligned}
$$

From the famous Banach's contraction mapping principle, we get that $P_{\mathscr{F}}(\gamma f+(I-A))$ has a unique fixed point, say $q \in H$, that is, $q=P_{\mathscr{F}}(\gamma f+(I-A))(q)$.

Step 6. We show that $\limsup _{n \rightarrow \infty}\left\langle\gamma f(q)-A q, x_{n}-q\right\rangle \leqslant 0$. To get this result, we choose a subsequence $\left\{x_{n_{i}}\right\} \subset\left\{x_{n}\right\}$ such that

$$
\limsup _{n \rightarrow \infty}\left\langle\gamma f(q)-A q, x_{n}-q\right\rangle=\limsup _{n \rightarrow \infty}\left\langle\gamma f(q)-A q, x_{n_{i}}-q\right\rangle .
$$

Correspondingly, there exists a subsequence $\left\{y_{n_{i}}\right\}$ of $\left\{y_{n}\right\}$. Since $\left\{y_{n_{i}}\right\}$ is bounded, there exists a subsequence $\left\{y_{n_{i}}\right\}$ of $\left\{y_{n_{i}}\right\}$ which converges weakly to $\omega$. Without loss of generality, we can assume that $y_{n_{i}} \rightarrow \omega$. Next, we will show that $\omega \in \mathscr{F}$.

Firstly, we prove $\omega \in E P(F)$. Since $y_{n}=T_{r_{n}} x_{n}$, we have $F\left(y_{n}, u\right)+\frac{1}{r_{n}}\left\langle u-y_{n}, y_{n}-x_{n}\right\rangle \geqslant 0$, for all $u \in C$. From $(C 2)$, we have $\left\langle u-y_{n}, \frac{y_{n}-x_{n}}{r_{n}}\right\rangle \geqslant F\left(u, y_{n}\right)$. It follows that,

$$
\left\langle u-y_{n_{i}}, \frac{y_{n_{i}}-x_{n_{i}}}{r_{n_{i}}}\right\rangle \geqslant F\left(u, y_{n_{i}}\right) .
$$

Since $\frac{y_{n_{i}}-x_{n_{i}}}{r_{n_{i}}} \rightarrow \infty, y_{n_{i}} \rightarrow \omega$ and (C4), we have $F(u, w) \leqslant 0$ for all $u \in C$. For $t \in(0,1]$ and $u \in C$, let $u_{t}=t u+(1-t) w$. Since $u \in C$ and $w \in C$, we have $u_{t} \in C$ and hence $F\left(u_{t}, w\right) \leqslant 0$. So, from (C1) and (C4), we have

$$
0=F\left(u_{t}, u\right) \leqslant t F\left(u_{t}, u\right)+(1-t) F\left(u_{t}, w\right) \leqslant t F\left(u_{t}, u\right) .
$$

That is, $F\left(u_{t}, u\right) \geqslant 0$. It follows from (C3) that $F(w, u) \geqslant 0$ for all $u \in C$ and hence $w \in \operatorname{EP}(F)$.

Secondly, since Hilbert spaces satisfy Opial's condition, from (3.16), we have

$$
\begin{aligned}
\liminf _{i \rightarrow \infty}\left\|y_{n_{i}}-\omega\right\|<\liminf _{i \rightarrow \infty}\left\|y_{n_{i}}-S w\right\| & =\liminf _{i \rightarrow \infty}\left\|y_{n_{i}}-S y_{n_{i}}+S y_{n_{i}}-S \omega\right\| \\
& \leqslant \liminf _{i \rightarrow \infty}\left\|S y_{n_{i}}-S w\right\| \\
& \leqslant \liminf _{i \rightarrow \infty}\left\|y_{n_{i}}-\omega\right\| .
\end{aligned}
$$

which derives a contraction. Thus, we have $\omega \in \mathscr{F}$.

Thirdly, we show $\omega \in \operatorname{VI}(B, C)$. Put

$$
\mathrm{T} \omega_{1}= \begin{cases}\mathrm{B} \omega_{1}+\mathrm{N}_{\mathrm{c}} \omega_{1}, & \omega_{1} \in \mathrm{C}, \\ \phi, & \omega_{1} \notin \mathrm{C} .\end{cases}
$$

From $B$ is relaxed $(\mu, v)$-cocoercive and condition (iv), we have

$$
(B x-B y, x-y) \geqslant(-\mu)\|B x-B y\|^{2}+v\|x-y\|^{2} \geqslant\left(v-\mu \lambda^{2}\right)\|x-y\|^{2},
$$

which yields that $B$ is monotone. Thus, $T$ is maximal monotone. Let $\left(\omega_{1}, \omega_{2}\right) \in G(T)$. Since $\omega_{2}-B \omega_{1} \in$ $\mathrm{N}_{\mathrm{C}} \omega_{1}$ and $\rho_{\mathrm{n}} \in \mathrm{C}$, we have

$$
\left\langle\omega_{1}-\rho_{n}, \omega_{2}-B \omega_{1}\right\rangle \geqslant 0
$$


On the other hand, from $\rho_{n}=P_{C}\left(I-s_{n} B\right) y_{n}$, we have

$$
\left\langle\omega_{1}-\rho_{n}, \rho_{n}-\left(I-s_{n} B\right) y_{n}\right\rangle \geqslant 0
$$

and hence

$$
\left\langle\omega_{1}-\rho_{n}, \frac{\rho_{n}-y_{n}}{s_{n}}+B y_{n}\right\rangle \geqslant 0
$$

It follows that

$$
\begin{aligned}
\left\langle\omega_{1}-\rho_{n_{i}}, \omega_{2}\right\rangle & \geqslant\left\langle\omega_{1}-\rho_{n_{i}}, B \omega_{1}\right\rangle \\
& \geqslant\left\langle\omega_{1}-\rho_{n_{i}}, B \omega_{1}\right\rangle-\left\langle\omega_{1}-\rho_{n_{i}}, \frac{\rho_{n_{i}}-y_{n_{i}}}{s_{n_{i}}}+B y_{n_{i}}\right\rangle \\
& =\left\langle\omega_{1}-\rho_{n_{i}}, B \omega_{1}-\frac{\rho_{n_{i}}-y_{n_{i}}}{s_{n_{i}}}-B y_{n_{i}}\right\rangle \\
& =\left\langle\omega_{1}-\rho_{n_{i}}, B \omega_{1}-B \rho_{n_{i}}\right\rangle+\left\langle\omega_{1}-\rho_{n_{i}}, B \rho_{n_{i}}-B y_{n_{i}}\right\rangle-\left\langle\omega_{1}-\rho_{n_{i}}, \frac{\rho_{n_{i}}-y n_{i}}{s_{n_{i}}}\right\rangle \\
& \geqslant\left\langle\omega_{1}-\rho_{n_{i}}, B \rho_{n_{i}}-B y_{n_{i}}\right\rangle-\left\langle\omega_{1}-\rho_{n_{i}}, \frac{\rho_{n_{i}}-y_{n_{i}}}{s_{n_{i}}}\right\rangle,
\end{aligned}
$$

which implies that $\left\langle\omega_{1}-\omega, \omega_{2}\right\rangle \geqslant 0$. We have $\omega \in \mathrm{T}^{-1} 0$ and hence $\omega \in \mathrm{VI}(\mathrm{B}, \mathrm{C})$. That is, $\omega \in \mathscr{F}$.

Finally, since $q=P_{\mathscr{F}}(\gamma f+(I-A))(q)$, we have

$$
\lim \sup _{n \rightarrow \infty}\left\langle\gamma f(q)-A q, x_{n}-q\right\rangle=\lim _{n \rightarrow \infty}\left\langle\gamma f(q)-A q, x_{n_{i}}-q\right\rangle=\langle\gamma f(q)-A q, w-q\rangle \leqslant 0 .
$$

Step 7. We will prove that $\left\{x_{n}\right\}$ and $\left\{y_{n}\right\}$ converge strongly to $q$.

$$
\begin{aligned}
\left\|x_{n+1}-q\right\|^{2}= & \left\|\alpha_{n}\left(\gamma f\left(x_{n}\right)-A q\right)+\beta_{n}\left(x_{n}-q\right)+\left(\left(1-\beta_{n}\right) I-\alpha_{n} A\right) S \rho_{n}\right\| \\
\leqslant & \left\|\beta_{n}\left(x_{n}-q\right)+\left(\left(1-\beta_{n}\right) I-\alpha_{n} A\right)\left(S \rho_{n}-q\right)\right\|^{2}+2 \alpha_{n}\left\langle\gamma f\left(x_{n}\right)-A q, x_{n+1}-q\right\rangle \\
\leqslant & {\left[\left\|\left(\left(1-\beta_{n}\right) I-\alpha_{n} A\right)\left(S \rho_{n}-q\right)\right\|+\left\|\beta_{n}\left(x_{n}-q\right)\right\|\right]^{2} } \\
& +2 \alpha_{n} \gamma\left\langle f\left(x_{n}\right)-f(q), x_{n+1}-q\right\rangle+2 \alpha_{n}\left\langle\gamma f(q)-A q, x_{n+1}-q\right\rangle \\
\leqslant & {\left[\left(1-\beta_{n}-\alpha_{n} \bar{\gamma}\right)\left\|x_{n}-q\right\|+\beta_{n}\left\|x_{n}-q\right\|\right]^{2}+2 \alpha_{n} \gamma \alpha\left\|x_{n}-q\right\|\left\|x_{n+1}-q\right\| } \\
& +2 \alpha_{n}\left\langle\gamma f(q)-A q, x_{n+1}-q\right\rangle \\
\leqslant & \left(1-\alpha_{n} \bar{\gamma}\right)^{2}\left\|x_{n}-q\right\|^{2}+\alpha_{n} \gamma \alpha\left[\left\|x_{n}-q\right\|^{2}+\left\|x_{n+1}-q\right\|^{2}\right] \\
& +2 \alpha_{n}\left\langle\gamma f(q)-A q, x_{n+1}-q\right\rangle
\end{aligned}
$$

which implies that

$$
\begin{aligned}
\left\|x_{n+1}-q\right\|^{2} \leqslant & \frac{1-2 \alpha_{n} \bar{\gamma}+\alpha_{n} \gamma \alpha}{1-\alpha_{n} \gamma \alpha}\left\|x_{n}-q\right\|^{2}+\frac{2 \alpha_{n}}{1-\alpha_{n} \gamma \alpha}\left\langle\gamma f(q)-A q, x_{n+1}-q\right\rangle \\
= & {\left[1-\frac{2(\bar{\gamma}-\gamma \alpha) \alpha_{n}}{1-\alpha_{n} \gamma \alpha}\right]\left\|x_{n}-p\right\|^{2}+\frac{\left(\alpha_{n} \bar{\gamma}\right)^{2}}{1-\alpha_{n} \gamma \alpha}\left\|x_{n}-q\right\|+\frac{2 \alpha_{n}}{1-\alpha_{n} \gamma \alpha}\left\langle\gamma f(q)-A q, x_{n+1}\right\rangle } \\
\leqslant & {\left[1-\frac{2(\bar{\gamma}-\gamma \alpha) \alpha_{n}}{1-\alpha_{n} \gamma \alpha}\right]\left\|x_{n}-q\right\|^{2} } \\
& +\frac{2(\bar{\gamma}-\gamma \alpha) \alpha_{n}}{1-\alpha_{n} \gamma \alpha} \times\left[\frac{\left(\alpha_{n} \bar{\gamma}^{2}\right) M_{2}}{2(\bar{\gamma}-\gamma \alpha)}+\frac{1}{\bar{\gamma}-\gamma \alpha}\left\langle\gamma f(q)-A q, x_{n+1}-q\right\rangle\right] \\
= & \left(1-\delta_{n}\right)\left\|x_{n}-q\right\|^{2}+\delta_{n} \sigma_{n},
\end{aligned}
$$

where

$$
M_{2}=\sup \left\{\left\|x_{n}-q\right\|^{2}: n \geqslant 1\right\}, \quad \delta_{n}=\frac{2(\bar{\gamma}-\gamma \alpha) \alpha_{n}}{1-\alpha_{n} \gamma \alpha}
$$


and

$$
\sigma_{n}=\frac{\left(\alpha_{n} \bar{\gamma}^{2}\right) M_{2}}{2(\bar{\gamma}-\gamma \alpha)}+\frac{1}{\bar{\gamma}-\gamma \alpha}\left\langle\gamma f(q)-A q, x_{n+1}-q\right\rangle .
$$

It is easy to see that $\delta_{n} \rightarrow 0, \sum_{n=1}^{\infty} \delta_{n}=\infty$ and $\limsup \sigma_{n} \leqslant 0$. Hence, by Lemma 2.4, the sequence $\left\{x_{n}\right\}$ converges strongly to $q$. Consequently, we can obtain that $\left\{y_{n}\right\}$ also converges strongly to $q$. The proof is complete.

\section{Application}

Theorem 4.1. Let $\mathrm{C}$ be a nonempty closed convex subset of a Hilbert space $\mathrm{H}$. Let $\mathrm{F}$ be a bifunction from $\mathrm{C} \times \mathrm{C}$ to $\mathfrak{R}$ which satisfies (C1)-(C4), let $\mathrm{S}$ be a nonexpansive mapping of $\mathrm{C}$ into $\mathrm{H}$ such that $\operatorname{Fix}(\mathrm{S}) \cap \mathrm{EP}(\mathrm{F}) \neq \phi$. Let $\mathrm{A}$ be a strongly positive linear bounded operator with coefficient $\bar{\gamma}>0$. Assume that $0<\gamma<\frac{\bar{\gamma}}{\alpha}$. Let $\mathrm{f}$ be a contraction of $\mathrm{H}$ into itself with a coefficient $\alpha(0<\alpha<1)$ and let $\left\{x_{n}\right\}$ and $\left\{y_{n}\right\}$ be sequences generated by $x_{1} \in \mathrm{H}$ and

$$
\begin{cases}F\left(y_{n}, u\right)+\frac{1}{r_{n}}\left\langle u-y_{n}, y_{n}-x_{n}\right\rangle \geqslant 0, & \forall u \in H, \\ \left.x_{n+1}=\alpha_{n} \gamma f\left(x_{n}\right)+\beta_{n} x_{n}+\left(\left(1-\beta_{n}\right) I-\alpha_{n} A\right)\right) S y_{n}, & \forall n \geqslant 1 .\end{cases}
$$

where $\left\{\alpha_{n}\right\} \subset[0,1]$ and $\left\{r_{n}\right\} \subset[0, \infty)$ satisfy

(i) $\lim _{n \rightarrow \infty} \alpha_{n}=0$ and $\sum_{n=1}^{\infty} \alpha_{n}=\infty$;

(ii) $0<\liminf _{n \rightarrow \infty} \beta_{n} \leqslant \limsup _{n \rightarrow \infty} \beta_{n}<1$;

(iii) $\liminf _{n \rightarrow \infty} r_{n}>0, \quad \lim _{n \rightarrow \infty}\left(r_{n+1}-r_{n}\right)=0$.

Then, both $\left\{x_{n}\right\}$ and $\left\{y_{n}\right\}$ converge strongly to $q \in \operatorname{Fix}(S) \cap E P(F)$, where $q=P_{\operatorname{Fix}(S) \cap E P(F)}(\gamma f+(I-A))(q)$, which solves the following variational inequality

$$
\langle\gamma f(q)-A q, p-q\rangle \leqslant 0, \quad \forall p \in \operatorname{Fix}(S) \cap E P(F) .
$$

Proof. Putting $\left\{s_{n}\right\}=0$ in Theorem 3.1, we can get the desired result easily.

Remark 4.2. If we take $\left\{s_{n}\right\}=0$ and $\beta_{\mathfrak{n}}=0$ in Theorem 3.1, we can get the results of Marino and $\mathrm{Xu}$ [8] and Plubtieng and Punpaeng [10] immediately.

Remark 4.3. If we take $\left\{s_{n}\right\}=0, \gamma=1, \beta_{n}=0$ and $A=I$ in Theorem 3.1, we can get the result of Takahashi and Takahashi [14] result immediately.

Theorem 4.4. Let $\mathrm{C}$ be a nonempty closed convex subset of a Hilbert space $\mathrm{H}$. Let $\mathrm{F}: \mathrm{C} \times \mathrm{C} \rightarrow \mathfrak{R}$ be a bifunction which satisfies (C1)-(C4), let $\mathrm{S}$ be a nonexpansive mapping of $\mathrm{C}$ into $\mathrm{H}$ and let $\mathrm{B}$ be a $\lambda$-Lipschitzian, relaxed $(\mu, v)$ cocoercive map of $\mathrm{C}$ into $\mathrm{H}$ such that $\mathscr{F}=\operatorname{Fix}(\mathrm{S}) \cap \mathrm{VI}(\mathrm{C}, \mathrm{B}) \neq \phi$. Let $\mathrm{A}$ be a strongly positive linear bounded operator with coefficient $\bar{\gamma}>0$ and assume that $0<\gamma<\frac{\bar{\gamma}}{\alpha}$. Let $\mathrm{f}$ be a contraction of $\mathrm{H}$ into itself with a coefficient $\alpha \in(0,1)$ and let $\left\{x_{n}\right\}$ be a sequence generated by $x_{1}=x \in \mathrm{H}$ and

$$
\left.x_{n+1}=\alpha_{n} \gamma f\left(x_{n}\right)+\beta_{n} x_{n}+\left(\left(1-\beta_{n}\right) I-\alpha_{n} A\right)\right) S P_{C}\left(I-s_{n}\right) P_{C} x_{n}, \forall n \geqslant 1,
$$

where $\left\{\alpha_{n}\right\} \subset[0,1]$ and $\left\{r_{n}\right\},\left\{s_{n}\right\} \subset[0, \infty)$ satisfy

(i) $\lim _{n \rightarrow \infty} \alpha_{n}=0$ and $\sum_{n=1}^{\infty} \alpha_{n}=\infty$;

(ii) $0<\liminf _{n \rightarrow \infty} \beta_{n} \leqslant \limsup _{n \rightarrow \infty} \beta_{n}<1$;

(iii) $\liminf _{n \rightarrow \infty} r_{n}>0, \lim _{n \rightarrow \infty}\left(r_{n+1}-r_{n}\right)=0$ and $\sum_{n=1}^{\infty}\left|s_{n+1}-s_{n}\right|<\infty$; 
(iv) $\left\{s_{n}\right\} \subset[a, b]$ for some $a, b$ with $0 \leqslant a \leqslant b \leqslant \frac{2\left(v-\mu \lambda^{2}\right.}{\lambda^{2}}$.

Then, $\left\{x_{n}\right\}$ converges strongly to $\mathrm{q} \in \mathscr{F}$, where $\mathrm{q}=\mathrm{P}_{\mathscr{F}}(\gamma \mathrm{f}+(\mathrm{I}-\mathrm{A}))(\mathrm{q})$, which solves the following variational inequality

$$
\langle\gamma f(q)-A q, p-q\rangle \leqslant 0, \forall p \in \mathscr{F} .
$$

Proof. Putting $F(x, y)=0$ for all $x, y \in C$ and $\left\{r_{n}\right\}=1$ for all $n$ in Theorem 3.1, then, we get $y_{n}=P_{C} x_{n}$, and we can obtain the desired conclusion easily.

\section{Acknowledgment}

This work was supported by the China Postdoctoral Science Foundation funded project (No. 2016M591468), the Social Science Foundation of Jilin Province (No. 2016BS13), the Education Department Foundation of Jilin Province (No. 2016068) and the Key Program of Jilin University of Finance and Economics (No. 2016Z10).

\section{References}

[1] E. Blum, W. Oettli, From optimization and variational inequalities to equilibrium problems, Math. Student, 63 (1994), 123-145. 2.1

[2] A. Bnouhachem, A modified projection method for a common solution of a system of variational inequalities, a split equilibrium problem and a hierarchical fixed-point problem, Fixed Point Theory Appl., 2014 (2014), 25 pages. 1

[3] L.-C. Ceng, A. Latif, A. E. Al-Mazrooei, Hybrid viscosity methods for equilibrium problems, variational inequalities, and fixed point problems, Appl. Anal., 95 (2016), 1088-1117. 1

[4] P. L. Combettes, S. A. Hirstoaga, Equilibrium programming in Hilbert spaces, J. Nonlinear Convex Anal., 6 (2005), 117-136. 2.2

[5] Q.-W. Fan, W. Wu, J. M. Zurada, Convergence of batch gradient learning with smoothing regularization and adaptive momentum for neural networks, SpringerPlus, 5 (2016), 1-17. 1

[6] H.-M. He, S.-Y. Liu, R.-D. Chen, Convergence results of multi-valued nonexpansive mappings in Banach spaces, J. Inequal. Appl., 2014 (2014), 12 pages. 1

[7] H. Iiduka, W. Takahashi, Strong convergence theorems for nonexpansive mappings and inverse-strongly monotone mappings, Nonlinear Anal., 61 (2005), 341-350. 1

[8] G. Marino, H.-K. Xu, A general iterative method for nonexpansive mappings in Hilbert spaces, J. Math. Anal. Appl., 318 (2006), 43-52. 1, 2.4, 4.2

[9] H. Piri, A general iterative method for finding common solutions of system of equilibrium problems, system of variational inequalities and fixed point problems, Math. Comput. Modelling, 55 (2012), 1622-1638. 1

[10] S. Plubtieng, R. Punpaeng, A general iterative method for equilibrium problems and fixed point problems in Hilbert spaces, J. Math. Anal. Appl., 336 (2007), 445-469. 1, 4.2

[11] R. T. Rockafellar, On the maximality of sums of nonlinear monotone operators, Trans. Amer. Math. Soc., 149 (1970), 75-88. 2

[12] Y.-S. Song, K. Promluang, P. Kumam, Y. J. Cho, Some convergence theorems of the Mann iteration for monotone $\alpha$ nonexpansive mappings, Appl. Math. Comput., 287/288 (2016), 74-82. 1

[13] T. Suzuki, Strong convergence of Krasnoselskii and Mann's type sequences for one-parameter nonexpansive semigroups without Bochner integrals, J. Math. Anal. Appl., 305 (2005), 227-239. 2.3

[14] S. Takahashi, W. Takahashi, Viscosity approximation methods for equilibrium problems and fixed point problems in Hilbert spaces, J. Math. Anal. Appl., 331 (2007), 506-515. 1, 4.3

[15] W. Takahashi, M. Toyoda, Weak convergence theorems for nonexpansive mappings and monotone mappings, J. Optim. Theory Appl., 118 (2003), 417-428. 1, 2

[16] Y.-H. Yao, Y. J. Cho, R.-D. Chen, An iterative algorithm for solving fixed point problems, variational inequality problems and mixed equilibrium problems, Nonlinear Anal., 71 (2009), 3363-3373. 1

[17] Y.-H. Yao, J.-C. Yao, On modified iterative method for nonexpansive mappings and monotone mappings, Appl. Math. Comput., 186 (2007), 1551-1558. 1

[18] Z.-C. Zhu, R.-D. Chen, Strong convergence on iterative methods of Cesro means for nonexpansive mapping in Banach space, Abstr. Appl. Anal., 2014 (2014), 6 pages. 1

[19] Z.-C. Zhu, B. Yu, A modified homotopy method for solving the principal-agent bilevel programming problem, Comput. Appl. Math., 2016 (2016), 26 pages. 1

[20] Z.-C. Zhu, B. Yu, Globally convergent homotopy algorithm for solving the KKT systems to the principal-agent bilevel programming, Optim. Methods Softw., 32 (2016), 69-85. 1 
[21] Z.-C. Zhu, B. Yu, Y.-F. Shang, A modified homotopy method for solving nonconvex fixed points problems, Fixed Point Theory, 14 (2013), 531-544. 1

[22] Z.-C. Zhu, B. Yu, L. Yang, Globally convergent homotopy method for designing piecewise linear deterministic contractual function, J. Ind. Manag. Optim., 10 (2014), 717-741. 1 